\title{
TranSVERSAL AND LONGITUDINAL IMAGES FROM THE RETINA OF THE LIVING EYE USING LOW COHERENCE REFLECTOMETRY
}

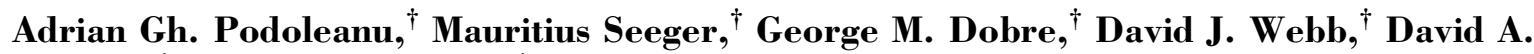 \\ Jackson, ${ }^{\dagger}$ and Fred W. Fitzke ${ }^{\ddagger}$ \\ University of Kent, Physics Laboratory, Applied Optics Group, Canterbury, CT2 7NR, United \\ Kingdom; Institute of Ophthalmology, Laboratory of Physiological Optics, London, United Kingdom \\ (Paper JBO/IB-009 received Aug. 12, 1997; revised manuscript received Oct. 27, 1997; accepted for publication Oct. \\ 30, 1997. )
}

\begin{abstract}
An optical coherence tomography (OCT) system to produce both longitudinal and transversal images of the in vivo human eye is presented. For the first time, OCT transversal images collected from the living eye at $50-\mu \mathrm{m}$ depth steps show details unobtainable with the state-of-the-art scanning laser ophthalmoscope. Images of up to $3 \times 3 \mathrm{~mm}$ are produced from the retina in less than a second. For images larger than $1.6 \times 1.6 \mathrm{~mm}$, a path modulation is introduced by the galvanometric scanning mirror and is used as an effective phase modulation method.๑ 1998 Society of Photo-Optical Instrumentation Engineers. [S1083-3668(98)01001-6]
\end{abstract}

Keywords optical coherence tomography; ophthalmology; imaging.

\section{INTRODUCTION}

Low coherence reflectometry ${ }^{1,2}$ is expected to become an increasingly widespread clinical tool for eye investigations. The technique has evolved rapidly in the past decade from initial investigations of different biological tissues ${ }^{3-7}$ to in vivo applications. ${ }^{8,9}$ Special attention has been given to applications in ophthalmology, ${ }^{10}$ with different setups for measurement of intraocular distances ${ }^{11-14}$ being devised and the technique being used to assess macula health. ${ }^{15}$ Lately, the emphasis has shifted steadily toward imaging, ${ }^{16}$ and this provides a useful complement to other approaches, e.g., ultrasonography, magnetic resonance, and scanning laser ophthalmoscopy. ${ }^{17}$

The technique has become known as optical coherence tomography (OCT) due to its capability to produce optical sections of the object under investigation. An OCT instrument for determining retinal thickness, which produces a longitudinal image of the eye in $1 \mathrm{~s}$ is already commercially available. ${ }^{18}$

OCT has been recently developed especially as an alternative investigative tool to the scanning laser ophthalmoscope (SLO), which provides transversal images from the eye. SLOs have been in use for at least a decade and have been used extensively clinically. Owing to the confocal principle involved and

Address all correspondence to A. G. Podoleanu. Fax: +44 1227 827558; E-mail: A. G. H. Podoleanu@ukc.ac.uk to the limitations imposed by the pupil diameter and the focal length of the eye lens, ${ }^{19}$ the depth accuracy of the commercially available SLOs is limited to about $300 \mu \mathrm{m}^{20}$ (although studies are being carried out to improve this ${ }^{21}$ ). The depth accuracy of the OCT images, however, is governed by the coherence length of the source, which could be as low as a few microns for tungsten lamps and Kerr lens mode-locked lasers, ${ }^{3,4}$ or a few tens of microns for the more practical superluminescent diodes (SLD). 6,10,11

The high depth resolution provided by low coherence interferometry is both an advantage and a disadvantage for the imaging process. In principle, there is a great potential for improving depth accuracy, as this depends on the source used and not on the object, which is the case with the SLO. However, the shorter the coherence length, the more sensitive the system is to eye movements and the easier it is disturbed by vibrations. Consequently, an acceptable tradeoff must be found between depth accuracy and ease of obtaining good and readily interpretable images of the eye.

Because the longitudinal images generated by the commercial OCT cannot be directly compared with the transversal images provided by the SLO, we investigated the possibility of using an OCT to deliver both longitudinal and transversal images.

We recently reported obtaining transversal OCT images from the living eye. ${ }^{22}$ In this paper we discuss the problems associated with the phase modu- 


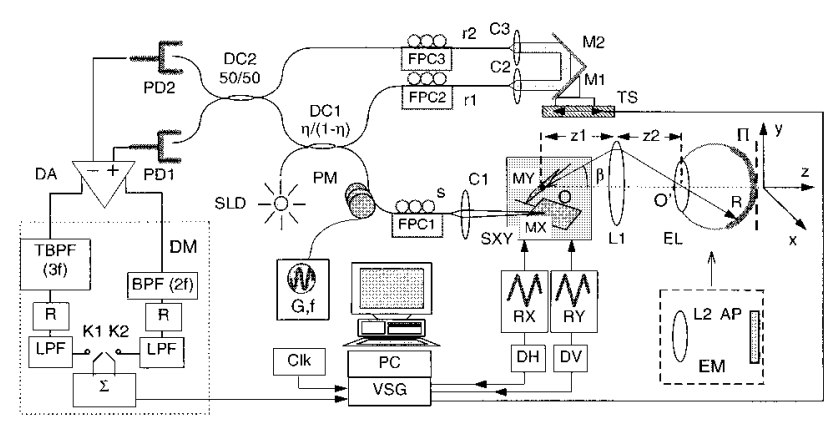

Fig. 1 Block diagram of the OCT system, SLD, superluminescent diode (Superlum SLD-361); C1, C2, C3, microscope objectives; DC1, DC2, directional couplers; PM, 5-cm-diameter piezocylinder with two turns of fiber acting as a phase modulator; $G$, sinusoidal generator, $f=30 \mathrm{kHz} ; M 1, M 2$, mirrors; L1, convergent lens; FPC 1,2,3, polarizer controllers; SXY, orthogonal scanning mirror pair; MX(MY), mirror of the $X(Y)$ scanner; DM, demodulation block; RX, RY, ramp generators; PD1, PD2, photodetectors; DA, differential amplifier (PD1, PD2, and DA are parts of a Nirvana New Focus balanced photodetector module); TBPF, tunable bandpass filter; $B P F$, bandpass filter; $R$, rectifier; LPF, low-pass filter; $\Sigma$ : analog adder; K1, K2, switches; DH, monostable circuit for the VSG line trigger; DV, monostable circuit for the VSG frame trigger; Clk, VSG clock generator; EL, eye lens; $R$, human retina; $E M$, eye model; L2, lens; AP, aluminum plate; $\Pi$, imaginary plane perpendicular to the optic axis and tangential to the retina; TS, computer-controlled translation stage; and PC, personal computer.

lation that appears when the age is scanned transversally and detail the conditions when this modulation can be effectively used as a carrier for the image signal. Transversal and longitudinal OCT images are shown from the living eye using this modulation only. We also present what we believe to be the first set of transversal OCT images from the retina with $50-\mu \mathrm{m}$ depth spacing.

\section{FUNCTIONAL OCT SYSTEM APPLICABLE TO THE LIVING EYE}

The configuration shown schematically in Figure 1 represents the current state of our OCT system. The core setup is based on the schematic diagram in Ref. 2. Light from a pigtailed superluminescent diode (SLD) central wavelength $\lambda=0.85 \mu \mathrm{m}$, is injected into a single-mode directional coupler (DC1), which transmits a percentage $\eta$ of the optical power into the object arm.

Light in the object arm propagates through a phase modulator (PM), which consists of two turns of fiber wrapped around a piezocylinder. Light subsequently travels via a microscope objective (C1), enters an orthogonal scanning mirror pair (SXY), and passes through a converging lens (L1, antireflection coated for $0.85 \mu \mathrm{m}, 6 \mathrm{~cm}$ diameter and $60 \mathrm{~mm}$ focal length), which brings the fan of rays to convergence at the eye lens. The reference beam, which emerges from port $\mathrm{r} 1$ of $\mathrm{DC} 1$, is of much higher power than the signal beam and is directed via microscope objectives $\mathrm{C} 2$ and $\mathrm{C} 3$ and mirrors M1 and M2 to the receiving coupler DC2, thus avoiding feedback to the SLD (it is known that SLDs are prone to oscillations and that their correlation profile suffers under back-reflections). The fiber end $\mathrm{r} 1$ is polished at a $10^{\circ}$ angle to reduce the noise associated with back-reflected light.

Mirrors M1 and M2 are mounted on a computercontrolled translation stage (TS), which can be moved continuously at a constant speed or discretely in steps $\geqslant 1 \mu \mathrm{m}$. Polarization controllers (FPC) are mounted in the sensing arm and in each of the reference arm ports.

The beam spot on mirror $\mathrm{MX}$, point $O$, situated at a distance $z_{1}$ in front of the lens L1, and the point $O$ where the rays enter the eye at a distance $z_{2}$ behind the lens L1, are conjugate points by virtue of L1. $O O^{\prime}$ defines the optic axis of the system, which coincides with the $z$ direction in the chosen system of coordinates (Figure 1). Two photodetectors, PD1 and PD2, collect the returned optical signals and their outputs are applied to the two inputs of a differential amplifier (DA) in a balanced detection configuration. The signal is then demodulated in the demodulator block (DM) and subsequently applied to a variable scan digital frame grabber (VSG).

Ramp generators $R X$ and $R Y$ drive the transversal scanners $\mathrm{X}$ and $\mathrm{Y}$ respectively, and also trigger signal acquisition by the frame grabber. DC1, FPC1, C1, SXY, and L1 are mounted on the chin rest used to accommodate the patient's head. A special mount was also devised for supporting different objects under investigation, such as the eye model (EM) in Figure 1, which consists of an aluminum plate diffuser (AP) placed at the back of lens L2. The system may be operated in three different regimes by altering the way the translation stage and the transversal scanner are driven and the demodulation block configured.

\subsection{TRANSVERSAL IMAGING}

In this regime, the galvo-scanning mirror pair SXY can be used to generate a frame, which could be of any shape (for example, a disk), in the plane perpendicular to the optic axis. In our experiments only conventional rectangular frames were produced, with the $X$ scanner driven by ramps of up to $F_{x}=600 \mathrm{~Hz}$ and the $\mathrm{Y}$ scanner driven by slower ramps, with a frequency $F_{y}=1.18 \mathrm{~Hz}$. The number of lines in the raster could easily be adjusted by controlling the frequency $F_{y}$.

Depending on the image size along the $x$ axis, which is governed by the amplitude $U$ of the signal applied to the $\mathrm{X}$ scanner, the demodulation block can be configured in two possible ways, will have discussed below. As explained in the next section, for values of $U$ less than $0.62 \mathrm{~V}$, a conventional phase modulation technique is employed. The piezocylinder is driven by a sinusoidal signal at frequency $f=30 \mathrm{kHz}$. Two bandpass filters (BPF) are used: the fixed frequency filter is tuned to $2 f$ and the tunable bandpass filter (TBPF) is tuned to $3 f$ 
and both switches $\mathrm{K} 1$ and $\mathrm{K} 2$ are closed. In this way, the residual intensity modulation from the phase modulator at $f$ is avoided. The amplitude of the driving signal applied to PM is adjusted to give the same modulation amplitude at $2 f=60 \mathrm{kHz}$ and $3 f=90 \mathrm{kHz}$ (this corresponds to an amplitude of the phase modulation of $\approx 3 \mathrm{rad}$ ). The two bandpass filter outputs are rectified (R) and low-pass filtered (LPF). An analog adder ( $\Sigma$ ) adds the two signals and the resulting sum is applied to the frame grabber. Since the demodulated signals at $2 f$ and $3 f$ have complementary variations in relation to the phase fluctuations, ${ }^{23}$ the amplitude of the demodulated signal after the adder is less sensitive to vibrations and thermal fluctuations.

For values of $U$ higher than $0.62 \mathrm{~V}$, only the tunable bandpass filter tuned to $90 \mathrm{kHz}$ is used, with $\mathrm{K} 1$ closed and K2 open. In this case, the modulation introduced by transversally scanning the target is employed, either on its own or in addition to piezomodulation (see Sec. 2.2).

\subsection{LONGITUDINAL IMAGING}

Longitudinal images can be collected in two different ways, depending on the translation stage speed:

1. Slow longitudinal scan imaging (SLI) with a slow-moving translation stage (TS) and a fast transversal scan across $\mathrm{X}$ or $\mathrm{Y}$. Each longitudinal scan results in an image in which the horizontal lines are given by either the $X$ or $Y$ scans at $600 \mathrm{~Hz}$. The modulation and demodulation configuration depend on the voltage applied to the galvo-scanner, as is the case in the transverse imaging (TI) regime;

2. Fast longitudinal scan imaging (DI), with the modulation carrier created by a Doppler shift. In this regime, the transversal scanner is run at a lower speed, or in fine steps. One complete run yields the image, while the lines are generated by repetitive movements of the translation stage. This regime was generally favored in OCT setups used for producing line scans. ${ }^{10,11}$ The tunable bandpass filter is tuned to the Doppler frequency, switch K1 is closed, and switch $\mathrm{K} 2$ is open.

\section{SIGNAL-TO-NOISE RATIO AND BANDWIDTH ANALYSIS}

The PD1 and PD2 photocurrents are given respectively by $i_{1}, i_{2}$ :

$$
\begin{aligned}
i_{1}= & \alpha P\{\eta(1-\eta) \mu E+(1-\eta)(1-\mu) R+2(1-\eta) \\
& \times[\eta \chi \mu(1-\mu) \mathrm{OR}]^{1 / 2}(\gamma(\mathrm{OPD})) \\
& \left.\times \cos \left(\varphi_{0}-\varphi_{r}-\frac{\pi}{2}\right)\right\}
\end{aligned}
$$

$$
\begin{aligned}
i_{2}= & \alpha P\{\eta(1-\eta)(1-\mu) E+(1-\eta) \mu R+2(1-\eta) \\
& \times[\eta \chi \mu(1-\mu) \mathrm{OR}]^{1 / 2}(\gamma(\mathrm{OPD})) \\
& \left.\times \cos \left(\varphi_{0}-\varphi_{r}+\frac{\pi}{2}\right)\right\}
\end{aligned}
$$

where $P$ is the SLD power, $\varphi_{0}$ signifies the phase in the sensing arm, and $\varphi_{r}$ is the phase in the reference arm. A phase shift of $\pi$ was assumed at each reflection (at the object and at mirrors M1 and M2). $O$ is the reflectivity of the object, $E$ is the reflectivity of the fiber end at port $s, \mu$ is the splitting ratio of coupler DC2, $\chi$ is the efficiency of coupling the object power back into port $\mathrm{s}$, and $R$ is the transfer efficiency of the reference beam from port $r 1$ to port $\mathrm{r} 2$, including the reflectivities of the mirrors M1 and M2 and the loss in coupling the light into port $\mathrm{r}_{2} . \alpha$ represents the responsivity of the photodetectors, which are assumed to be identical, and $\gamma(\mathrm{OPD})$ represents the modulus of the correlation function of the field for an optical path difference (OPD). In (1) and (2) we have considered $R \gg E \gg O$. After subtraction of the photodetected signals, the differential amplifier delivers a signal proportional to

$$
\begin{aligned}
\Delta i= & \alpha P\{\eta(1-\eta)(2 \mu-1) E+(1-\eta)(1-2 \mu) R+4(1 \\
& \left.-\eta)[\eta \chi \mu(1-\mu) \mathrm{OR}]^{1 / 2}(\gamma(\mathrm{OPD})) \sin \left(\varphi_{0}-\varphi_{r}\right)\right\} .
\end{aligned}
$$

Let us suppose that DC2 is balanced, $\mu=0.5$, and consequently the first two terms which represent the contribution of the bias are eliminated. In this balanced case, ${ }^{24}$ the mean-square current fluctuation, $\left\langle\Delta I_{n}^{2}\right\rangle$, is expressed as

$$
\left\langle\Delta I_{n}^{2}\right\rangle=2 e B\left[\left\langle I_{\text {ref }}\right\rangle+\left\langle I_{E}\right\rangle\right]+4 B \lambda^{2} \frac{\left\langle I_{\text {ref }}\right\rangle\left\langle I_{E}\right\rangle}{c \Delta \lambda}
$$

where $e$ is the electron charge, $B$ is the electrical bandwidth, $\Delta \lambda$ is the optical line width, and $I_{\text {ref }}$ and $I_{E}$ in the total current (PD1 and PD2) owing to the reference optical signal and the back-reflection from the fiber end $s$, respectively.

When

$$
\left\langle I_{E}\right\rangle \ll \frac{e c \Delta \lambda}{2 \lambda^{2}}=0.664 \mu A,
$$

the first term in (4) dominates, i.e., the system operates in the shot noise-limited regime [use was made of $I_{E} \ll I_{\text {ref }}$ and a value of $\Delta \lambda=20 \mathrm{~nm}$ was taken in (4)]. Using the first two terms in (1) and (2), considering $E=3.5 \%$, with $\eta=25 \%$, and $\alpha$ $=0.5 \mathrm{~mA} / \mathrm{mW}$ (silicon), inequality (5) gives an upper limit for the source power $P_{\mathrm{SNL}}=202 \mu \mathrm{W}$. Higher-power values would be permitted if the fiber end reflectivity $E$ was smaller. Polishing the fiber at an angle lowers the end reflection but at the same time reduces the efficiency of coupling the light back into the system. We favored having a higher collection efficiency from the object and thus 
only angle polished the fiber end in port r1. Taking into account that in the simplified model we neglected losses in the couplers and thermal and electronic noise, we increased the power to $360 \mu \mathrm{W}$, in which case the excess photon noise is slightly more than the shot noise and the power incident at the eye is $90 \mu \mathrm{W}$. This level of power is similar to that used by other research groups ${ }^{14,16}$ and is below the $400-\mu \mathrm{W}$ level for 100-s irradiation permitted by laser safety regulations (Euronorm EN 60825-1) at wavelengths in the 800-nm band.

Evaluating the signal-to-noise ratio in the manner described in Ref. 24, we obtain (for $\mu=0.5$ )

$$
\frac{S}{N}=\frac{\alpha O \chi(1-\eta) \eta P}{e B\left[1+2 \lambda^{2} \alpha \eta(1-\eta) E P /(e c \Delta \lambda)\right]} .
$$

The size $d$ of a pixel defined on the retina (or at the back of lens L2 in Figure 1) is the diffractionlimited spot size for a diameter of a beam $D$ focused by a lens of focal distance $f_{2}$ and is given by

$$
d=1.22 \lambda \frac{f_{2}}{D} .
$$

Taking into account the number of pixels $N_{x}$ that constitute a line scan of length $\Delta X$ in the imaginary plane $\Pi$ in Figure 1, the image bandwidth can be evaluated as

$$
B_{i}=N_{x} \frac{1}{2 T_{x}}=\frac{\Delta X}{d} \frac{1}{2 T_{x}}=0.41 \frac{D}{\lambda} \frac{\Delta X}{T_{x} f_{2}},
$$

where $T_{x}$ represents the time taken to scan a line. This bandwidth will arise on either side of the carrier, since the carrier is modulated in intensity by variations in image reflectivity.

Taking into account the scanner sensitivity $k$, the amplitude of the driving ramp generating a scan of span $\Delta X$ is obtained as

$$
U=\frac{z_{2}}{z_{1}} \frac{\Delta X}{2 k f_{2}} .
$$

In order to get maximum speed out of the $X$ scanner and obtain a 512-line image in less than $1 \mathrm{~s}$, the scanner is driven at a frequency $F_{x}$ higher than the rolloff frequency, in which case $k$ depends on $F_{x}$, and higher values of $F_{x}$ result in smaller values of $k$. For example, for $F_{x}=600 \mathrm{~Hz}, k=45.38 \mathrm{mrad} / \mathrm{V}$ (which is 1.54 times smaller than at $10 \mathrm{~Hz}$ ) and the galvo-scanner flyback time is about $20 \%$ of the line period. We have eliminated the display of an image during flyback by using a monostable circuit $(\mathrm{DH})$ with a delay of $340 \mu$ s to trigger the line display of the grabber. Consequently, the duration of a line scan is $T_{x}=1.33 \mathrm{~ms}$.

The carrier frequency $f$ should be chosen in such a way that the bandwidth about $2 f$ does not overlap with the bandwidth about $3 f$, which implies that

$$
f>2 B_{i} .
$$

Since our PM is limited to $30 \mathrm{kHz}$ (although higher PM piezomodulation frequencies have been reported $\left.^{8}\right), B_{i}$ should be less than $15 \mathrm{kHz}$. Using Eqs. (8) and (9), for $\lambda=0.85 \mu \mathrm{m}, D=2 \mathrm{~mm}, z_{2}$ $=10.2 \mathrm{~mm}, z_{1}=14.5 \mathrm{~mm}$, and $f_{2}=20 \mathrm{~mm}$, a bandwidth of $15 \mathrm{kHz}$ would limit the size of the image to $\Delta X=0.42 \mathrm{~mm}\left(N_{x}=40\right.$ pixels $)$ and the amplitude of the $\mathrm{X}$ scanner voltage to $U_{1}=0.16 \mathrm{~V}$.

For higher $\Delta X$ values, higher carrier frequencies and values for $B$ are required. In order to limit the distortion of the deflected beam due to the size of lens L1, we limited the amplitude applied to the scanner to $U_{M}=1.3 \mathrm{~V}$, resulting in a span $\Delta X$ $=3.35 \mathrm{~mm}$ [from Eq. (9)]. Consequently, the value of $B_{i}$ goes up to $121 \mathrm{kHz}$ [from Eq. (8)], and the $30-\mathrm{kHz}$ modulation cannot act as a carrier. For images of larger sizes, therefore, in the absence of a faster device, a different method of providing phase modulation has to be used.

We recently proposed and investigated a technique for achieving fast modulation rates that is based on the phase modulation introduced by the galvo-scanner itself, ${ }^{25}$ and this technique was applied here to obtain images of larger sizes. We showed that at coherence the object is sampled with a sampling function whose Newton rings' appearance is a consequence of a quadratic OPD dependence on the deflection angle $\beta$. When the beam is scanned across the sampling function, the photodetected signal is modulated at a frequency $\nu$. The maximum value of this frequency $\nu_{g}$, is expressed as

$$
\nu_{g}=\frac{4 k U}{\lambda T_{x}} f_{2} \frac{z_{1}^{2}}{z_{2}^{2}}\left|\beta_{m}\right|
$$

where $\left|\beta_{m}\right|$ is the maximum angle for which interference still occurs. Interference signals are produced for $|\mathrm{OPD}|<l_{c}$, where $l_{c}$ is the coherence length of the source, and the angle $\beta$ for which $|\mathrm{OPD}|=l_{c}$ is $\beta_{c}$ :

$$
\beta_{c}=\sqrt{\frac{l_{c}}{f_{2}}} \frac{z_{2}}{z_{1}} .
$$

For $|\beta|>\beta_{c},\left|\beta_{m}\right|=\beta_{c}$ and for $|\beta|<\beta_{c},\left|\beta_{m}\right|=k U$. According to (11), this means that for $U<U_{c}$ $=\beta_{c} / k, \nu_{g}$ has a quadratic dependence on $U$, and for $U>U_{c}$, the dependence becomes linear. This can be understood by the fact that for $|\beta|$ values above $\beta_{c}$, the explored areas correspond to $|\mathrm{OPD}|$ values outside coherence and consequently they do not contribute to the interference signal. Up to $\beta_{c}$, interference is obtained during the entire duration of a transversal scan, $T_{x}$. Above $\beta_{c}$, the time interval during which interference takes place and the period $1 / \nu$ of the modulation signal are propor- 


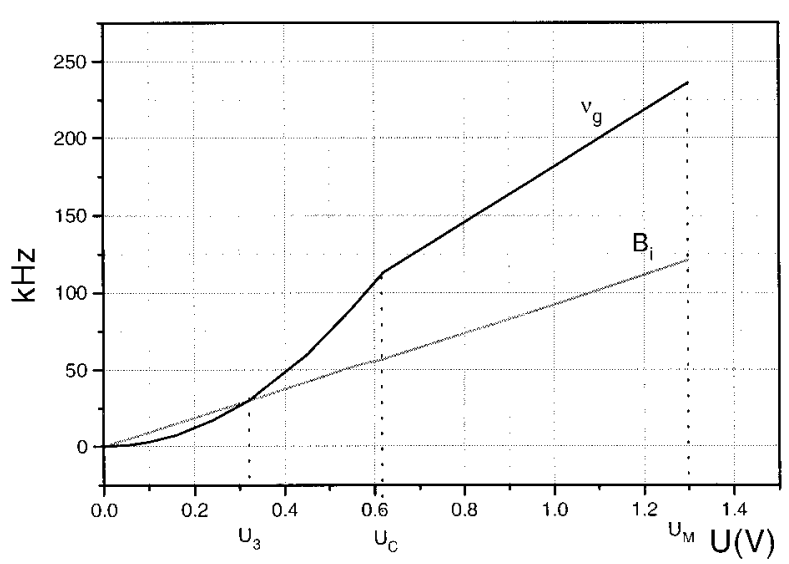

Fig. 2 The frequency $\nu_{g}$ due to galvo-scanner modulation and the image bandwidth, $B_{i}$ against the amplitude of the driving signal, $U$.

tional to $\beta_{C} / \beta$ and both decrease with increasing $U$. As a result, the dependence $\nu_{g}(U)$ becomes linear.

For $U>U_{C}$, components up to $\nu_{g}$ are generated [from (11) and (12)]:

$$
\nu_{g}=4 k \frac{U}{T_{x}} \frac{z_{1}}{z_{2}} \frac{\sqrt{f_{2} l_{c}}}{\lambda}
$$

With $l_{c}=31.9 \mu \mathrm{m}$ and the numerical values above, $U_{C}=0.62 \mathrm{~V}$ and for $U_{M}=1.3 \mathrm{~V}, \nu_{g}=236 \mathrm{kHz}$.

The graphs in Figure 2 show the dependence of $\nu_{g}$ and $B_{i}$ on $U$. Table 1 displays the numerical values for the $\Delta X$ span size at the back of lens L2 of a 2 -cm focal length, and $B_{i}$ and $\nu_{g}$ in relation to the voltage applied to the $X$-scanner. $\Delta R$ represents the gap between adjacent Newton rings at the edge of the sampling function for each amplitude $U$. This spacing places a minimum value on the size of features that can be resolved in the image.

To illustrate the galvo-scanner modulation experimentally, we used the arrangement denoted by

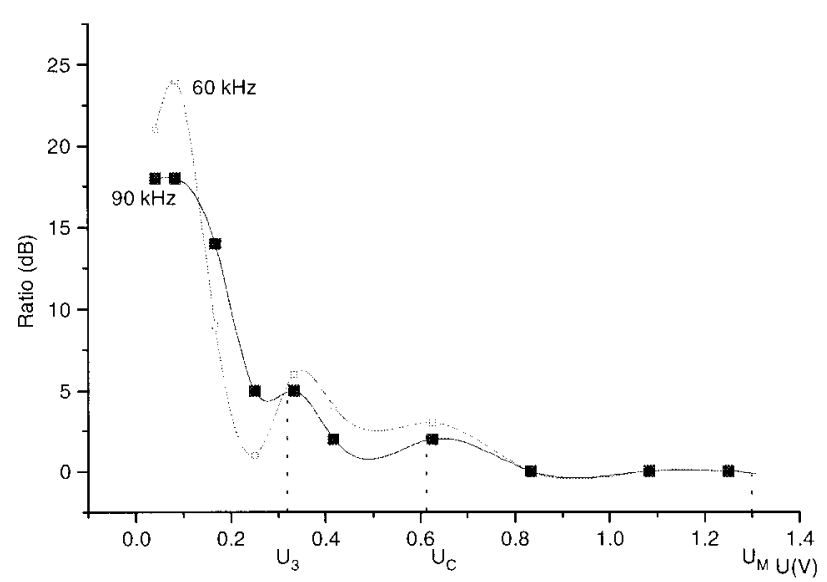

Fig. 3 Ratio of the amplitudes of the $60-\mathrm{kHz}$ and $90-\mathrm{kHz}$ components in the photodetected signal spectrum with and without the sinusoidal PM modulation, versus the amplitude of the ramp driving signal, $U . F_{x}=600 \mathrm{~Hz}$, the $Y$ galvo-scanner not driven.

EM in Figure 1. The $X$ scanner was driven by ramp signals of frequency $F_{x}=600 \mathrm{~Hz}$ and different amplitudes $U$. The $Y$ scanner was not driven. Figure 3 shows the ratio between the amplitudes of the components in the spectrum of the photodetected current in a bandwidth of $6 \mathrm{kHz}$ about $60 \mathrm{kHz}$ (i.e., $2 f$ ) and in a bandwidth of $9 \mathrm{kHz}$ about $90 \mathrm{kHz}$ (i.e., $3 f$ ) obtained with and without the sinusoidal PM modulation at $30 \mathrm{kHz}$. The amplitudes were averaged over 100 measurements. These graphs show that for voltages higher than $U_{C}=0.62 \mathrm{~V}(\Delta X$ $=1.6 \mathrm{~mm}$ ), the PM modulation does not have any noticeable effect on the demodulated signal, $\nu_{g}$ acting as an efficient carrier. Little enhancement is brought about by $\mathrm{PM}$ in the range $U_{3}$ to $U_{\mathrm{C}}$. For $U<U_{3}=0.32 \mathrm{~V}, \nu_{g}<B_{i}$ and $\nu_{g}$ cannot act as a carrier. Consequently, for $U<U_{3}$, the modulation introduced by PM becomes more important, as proved by the graphs in Figure 3. The modulation

Table 1 Numerical values for $\Delta X$.

\begin{tabular}{lllccc}
$\begin{array}{l}\text { Key bandwidth values on } \\
\text { the horizontal axis of the } \\
\text { graphs in Fig. 2. }\end{array}$ & $U(\mathrm{~V})$ & $\Delta X(\mathrm{~mm})$ & $B_{i}(\mathrm{kHz})$ & $\nu_{g}(\mathrm{kHz})$ & $\begin{array}{c}\Delta R(\mu \mathrm{m}) \\
\text { (Newton } \\
\text { rings) }\end{array}$ \\
\hline $1\left(B_{i}=15 \mathrm{kHz}\right)$ & $U_{1}=0.16$ & 0.416 & 15 & 7.5 & 58 \\
$2\left(B_{i}=22.5 \mathrm{kHz}\right)$ & $U_{2}=0.24$ & 0.62 & 22.5 & 17 & 39 \\
$3\left(B_{i}=\nu_{g}\right)$ & $U_{3}=0.32$ & 0.83 & 30 & 30 & 29 \\
$4\left(\nu_{g}=60 \mathrm{kHz}\right)$ & $U_{4}=0.45$ & 1.16 & 42 & 60 & 21 \\
$5\left(\nu_{g}=90 \mathrm{kHz}\right)$ & $U_{5}=0.55$ & 1.43 & 52 & 90 & 17 \\
$C\left(\nu_{g}\right.$ becomes linear in $\left.U\right)$ & $U_{C}=0.62$ & 1.6 & 57 & 113 & 15 \\
$M($ max voltage applied) & $U_{M}=1.3$ & 3.35 & 121 & 236 & 7 \\
\hline \hline
\end{tabular}


is essential for the case $U<U_{1}$, when (10) is satisfied and $\nu_{g} \ll B_{i}<15 \mathrm{kHz}$.

When $U>U_{C}$, we use the tunable bandpass filter tuned to $90 \mathrm{kHz}$ and operating at $Q=2$ to give a value of $B=45 \mathrm{kHz}$. Higher bandwidth values are not possible owing to the differential amplifier in the balance photodetection block (New Focus, 125 $\mathrm{kHz}$ ). This means that we have to sacrifice image bandwidth, which reduces transversal resolution. Consequently, the pixel size on the target is increased from the value given by (7) $(d=10.4 \mu \mathrm{m})$ to an effective value $d_{\text {eff }}$. Taking into account that the receiver acts as a low-pass filter of bandwidth $B / 2$, thereby lowering the processed signal bandwidth from $B_{i}$ to $B / 2$, the effective pixel size is $d_{\text {eff }}$ $=\left(2 B_{i} / B\right) d=56 \mu \mathrm{m}$. For the maximum image size of $3.35 \mathrm{~mm}$ imposed above (when $U=U_{M}=1.3 \mathrm{~V}$ ), the corresponding number of pixels is $N_{x}=60$, although this is a very conservative calculation. When $\nu_{g}=90 \mathrm{kHz}$ (point 5 in Table 1), it can be shown ${ }^{25}$ that the gap $\Delta R$ between adjacent Newton rings is $17 \mu \mathrm{m}$, which allows sampling of pixels down to $34 \mu \mathrm{m}$. It can be stated without going into a more detailed analysis that the pixel size varies between 34 and $56 \mu \mathrm{m}$ across the target, depending on the inclination of different object features relative to the coherence wavefront.

The diffraction-limited pixel size given by (7) could be achieved for $U=U_{M}$ and $B=45 \mathrm{kHz}$ when the $X$ scanner is driven at line rates below $111 \mathrm{~Hz}$. In this case, the time taken to produce a frame increases proportionally with $1 / F_{x}$.

The total time to produce a frame for the transversal imaging regime is

$$
T_{T}=\frac{N_{y}}{F_{x}}
$$

with $N_{y}$ the number of lines in the frame. At $F_{x}$ $=600-\mathrm{Hz}$ line rate, we produce an image of 768 $\times 512$ pixels in $T_{T}=0.85 \mathrm{~s}$.

For a given bandwidth $B$, the minimum signal that could be detected by the system is obtained from (6) for a signal-to-noise ratio of 1 . For the example considered above, with $\chi=0.4, P=360 \mu \mathrm{W}$, and $B=45 \mathrm{kHz}$, a minimum detectable value of the object reflectivity $O_{\min }=-90.2 \mathrm{~dB}$ is obtained. When $U<0.16 \mathrm{~V}$, the quality factor $Q$ of the bandpass filters could be increased to enhance the signal-to-noise ratio.

For longitudinal images in the SLI regime, the bandwidth and the minimum detectable signal are the same as in the TI case. Different speeds can be used. We decided to obtain an image in $0.8 \mathrm{~s}$ (approximately equal to $T_{T}$, which allows the use of the same VSG settings for longitudinal imaging as for transversal imaging). In order for the translation stage to gain speed, the image is produced after a 0.2 -s delay introduced by the monostable circuit (DV). The clock signal controlling VSG is the same as the one used in the TI regime, so the range $\Delta Z(\mathrm{~mm})$ displayed (the frame height) is $0.8 \mathrm{~s}$ multiplied by the speed $(\mathrm{mm} / \mathrm{s})$. The translation stage speed should be limited to a value such that during the time taken to produce a transversal line, $1 / F_{x}$, the reference path is modified by less than half the longitudinal pixel size, which is about half of the coherence length of the source, $l_{c}$

$$
v_{\max }=\frac{l_{c}}{4} F_{x}=4.78 \mathrm{~mm} / \mathrm{s} .
$$

For this value, it can be shown that the Doppler frequency is much less than the bandwidth $B_{i}$, so the tunable bandpass filter in the demodulation block does not need retuning. Depending on the depth size $\Delta Z$, the number of pixels $N_{z}$ is given by

$$
N_{z}=\frac{2 \Delta Z}{l_{c}} .
$$

For longitudinal images in the DI regime, VSG displays the object $z$ axis along the horizontal direction and the object $x$ or $y$ axis along the vertical direction. For this regime, devices for fast longitudinal OPD variation are needed. ${ }^{8,26}$ Since our translation stage has a limited speed, resulting in too long a time to produce a frame, we do not use this regime for imaging but only for producing a coherence reflectogram along the $z$ axis. Such a system is discussed in Ref. 11.

\section{AdJuStMENT AND IMAge SETTINGS: WAVEFRONT DISTORTION}

The transversal images collected from the eye need correction owing to the curvature of the coherence surface at the back of the eye lens. To illustrate this, we used the EM arrangement in Figure 1. We applied $1.3-\mathrm{V}$ amplitude ramps on both galvoscanners and shifted the translation stage in 24 steps of $5 \mu \mathrm{m}$. The transversal images evolve from a disk through circles of expanding radius when the reference path is increased, as shown by four of the images presented in Figure 4(a). Using a computer program, we obtained the pseudolongitudinal image shown in Figure 4(b). For any $(x, y)$ pixel in the image, the program calculates the amplitudes of the coherence signal for all 24 depth positions. In Figure $4(\mathrm{~b})$, this dependence on $z$ is shown for all the pixels $(384, y)$ with $y$ from 1 to 512 . A similar image was obtained in the SLI regime by moving the translation stage at a speed $v=0.35 \mathrm{~mm} / \mathrm{s}$ over 280 $\mu \mathrm{m}$.

Figures 4(a) and 4(b) clearly show that the longitudinal slice looks curved. This curvature has to be taken into account when imaging the retina. With our system at the extremity of the target (i.e., for the ray deflected when the driving voltage is at a maxi- 


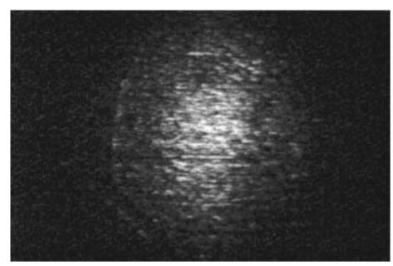

0 microns

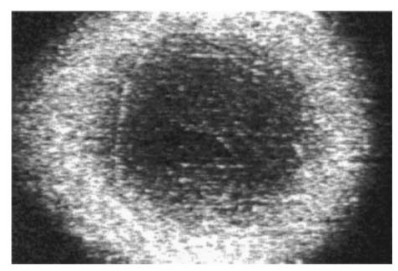

50 microns

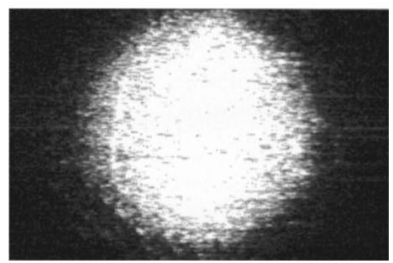

25 microns

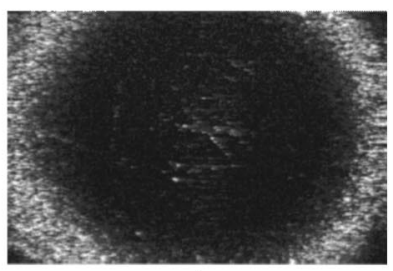

75 microns (a)

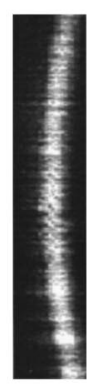

(b)

Fig. 4 (a) Images collected from the aluminum plate in the T1 regime for four different positions of the translation stage. Amplitude of the ramp voltage applied to both scanners: $1.3 \mathrm{~V}$ (raster size of 3.35 by $3.35 \mathrm{~mm}$ on the aluminum plate); modulation imprinted by the galvo-scanner only, $F_{x}=600 \mathrm{~Hz}, T_{y}=0.85 \mathrm{~s}$. (b) Software section in depth in the set of 24 images collected in $5-\mu \mathrm{m}$ steps. Vertical: 512 pixels corresponding to $\Delta Y=3.35 \mathrm{~mm}$; section taken in the middle of images at the position $X=0$ (384th pixel); Horizontal: $120 \mu \mathrm{m}, 24$ pixels.

mum), half of the OPD is about $\epsilon=70 \mu \mathrm{m}$ greater than for the central ray. This value is consistent with $\epsilon$ given by

$$
\boldsymbol{\epsilon}=f_{2}\left[1-\cos \left(\frac{z_{1}}{z_{2}} k U\right)\right]
$$

for $U=1.3 \mathrm{~V}$ and $f_{2}=2 \mathrm{~cm}$. This distortion is due to the particular optics used, with the fan of rays convergent on the eye. Had a telecentric optical system been used, the surface reconstructed in the longitudinal image would appear as a plane. Unfortunately, a telecentric system is not applicable to the eye, owing to the small aperture available.

\section{OCT IMAGES OF THE RETINA}

All the images presented here were collected with $90 \mu \mathrm{W}$ of optical power incident on the eye. No averaging and no enhancement procedures were applied. The images have a size of 768 $\times 512$ pixels. The intensity of each pixel represents the 8-bit digital value of the analog signal delivered by the demodulation block.

\subsection{EN-FACE IMAGES AT DIFFERENT DEPTHS}

There are reports in the literature on the production of tomographic sections of the retina using the SLO. ${ }^{27}$ OCT can provide such images at steps one order of magnitude lower.

Batches of 30 en-face images were collected from the living eye at different $Z$ depths obtained by stepping the translation stage by $50 \mu \mathrm{m}$ every $1 \mathrm{~s}$. After storage, the images were inspected and the batches showing disturbances due to eye movement were discarded. Figure 5(a) shows six images retained from a larger batch. The higher the distance quoted underneath each image, the deeper is the penetration into the retina and the eye. Pixel brightness is proportional to the amplitude of the coherence signal. From the fourth to the fifth image, the depth difference is $100 \mu \mathrm{m}$ because during the collection of the image for the intermediate step, the subject blinked and the image was discarded. There are noticeable differences between the images, with different details coming into and out of coherence as the reference path is scanned. Owing to the curvature of the retina, fragments of retinal features are visible in a $400-\mu \mathrm{m}$ longitudinal range. These images show that the blood vessel that appears dark in the last image in Figure 5(a) is about 200 $\mu \mathrm{m}$ thick (taking into account a value of 1.4 as an average for the index of refraction of the retinal tissue).

Another batch of transversal images from a different volunteer is shown in Figure 5(b). In this case the eye was oriented so that when the transversal scanners were not driven the patient could see the spot in the center of his visual field, thus bringing the foveal pit into the center of the image.

\subsection{LONGITUDINAL IMAGES}

We collected images of the same eye in the SLI regime using the same orientation as in Figure 5(b). A set of four images is presented in Figure 6, with $v$ $=0.625 \mathrm{~mm} / \mathrm{s}$ for the top images and 1 and $2 \mathrm{~mm} / \mathrm{s}$ for the bottom images, producing a frame of height $\Delta Z=0.5,0.8$, and $1.6 \mathrm{~mm}$, respectively. The characteristic foveal pit can be easily seen. For the images at the bottom of Figure 6 obtained with a 1.3-V amplitude signal applied to the $X$ scanner $(\Delta X$ $=3.35 \mathrm{~mm}$, minimum 60 pixels), only galvoscanner-induced phase modulation was employed. For the images at the top of Figure 6 obtained with $U=0.32 \mathrm{~V}(\Delta X=0.83 \mathrm{~mm})$ modulation to the PM was used to enhance the signal. In this last case, $B_{i}=30 \mathrm{kHz}$, so that the pixel size was about $d_{\text {eff }}$ $=(60 / 45) d=14 \mu \mathrm{m}$; i.e., there were $N_{x}=59$ pixels. The vertical pixel size was about $l_{c} / 2=16 \mu \mathrm{m}$; i.e., 


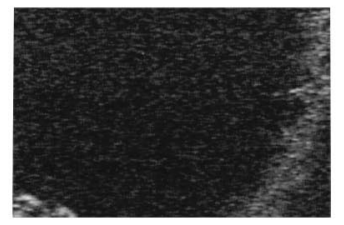

o microns

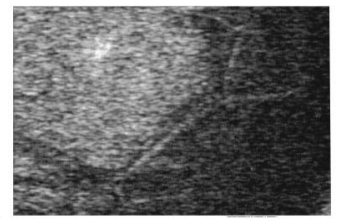

100 microns

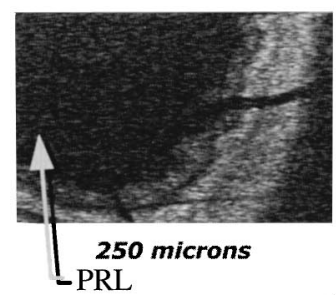

(a)

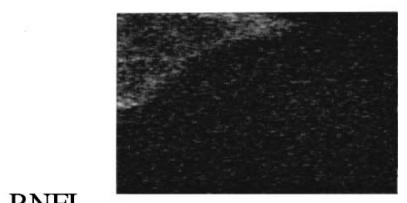

RNFL

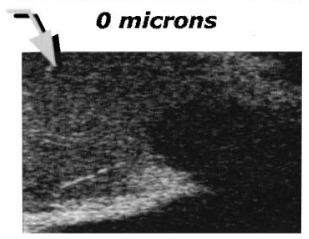

100 microns

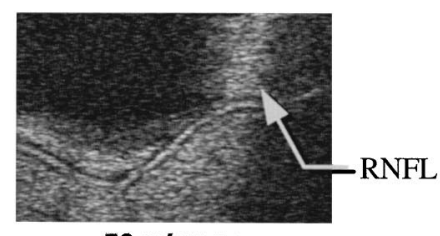

50 microns

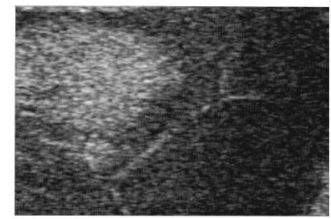

150 microns

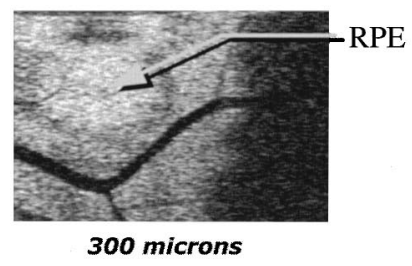

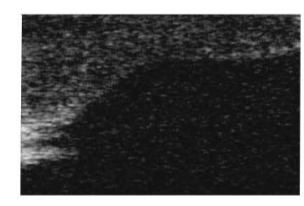

50 microns

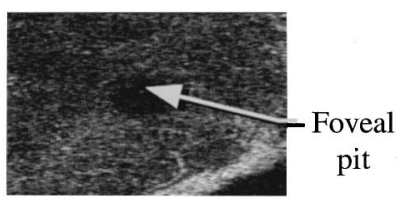

150 microns (b)

Fig. 5 Transversal images collected from the left eye of two volunteers at $50 \mu \mathrm{m}$ steps in depth. The size of the image is approximated at $\Delta X=3.35 \mathrm{~mm}$ by $\Delta Y=2.57 \mathrm{~mm}$ for $1.3-V$ amplitude applied on the $X$ galvo-scanner and $1 \mathrm{~V}$ applied on the $Y$ galvoscanner (considering an eye lens of $2 \mathrm{~cm}$ focal length); $F_{x}$ $=600 \mathrm{~Hz}, T_{y}=0.85 \mathrm{~s}$. (a) Going through a blood vessel. (b) Displaying the foveal pit. RNFL, retinal nerve fiber layer; PRL, photoreceptor layer (minimal backscatter signal); RPE, retinal pigment epithelium.

there were $N_{z}=31$ vertical pixels in the two top images, 50 in the bottom left image, and 100 in the bottom right image.

Figure 7 shows the images at the bottom of Figure 6 corrected for the wavefront distortion in Figure $4(\mathrm{~b})$, using the equation:

$$
\operatorname{correction}\left(X_{\text {pixel }}\right)=\frac{\epsilon}{512 \Delta Z}\left(X_{\text {pixel }}-384\right)^{2},
$$

where $\epsilon$ is $70 \mu \mathrm{m}$ and $\Delta Z$ is 0.8 and $1.6 \mathrm{~mm}$, respectively. This is only an approximate correction, which depends mainly on the estimated focal
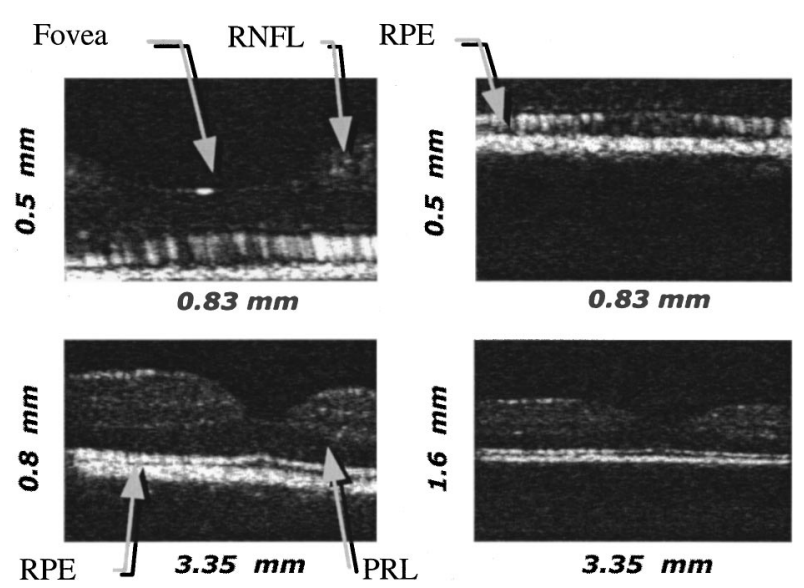

Fig. 6 Longitudinal images of the retina for the eye in Figure 5(b) for different depth ranges $\Delta Z$ as shown on each picture, taken with different speeds $v(\mathrm{~mm} / \mathrm{s})=\Delta Z \mathrm{~mm} /(0.8 \mathrm{~s})$. (To obtain the geometric thickness, $\Delta Z$ must be divided by the index of refraction, $\approx 1.4$.) $Y$ scanner not driven. Top images: $U=U_{3}=0.32 \mathrm{~V}$, PM modulated; bottom images: $U=U_{M}=1.3 \mathrm{~V}, \mathrm{PM}$ not modulated.

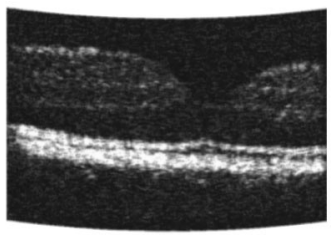

$3.35 \mathrm{~mm} \times 0.8 \mathrm{~mm}$

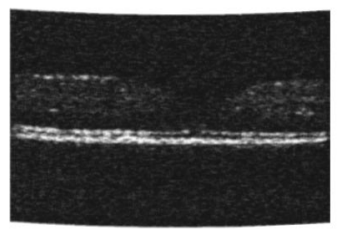

$3.35 \mathrm{~mm} \times 1.6 \mathrm{~mm}$
Fig. 7 Images in the bottom of Figure 6 corrected for the wavefront tilt in Figure 4(b).

length of the eye measured. The value of $\epsilon$ in (18) is determined on the basis of this focal length.

\section{CONCLUSIONS}

The paper describes the implementation of a multifunctional OCT for clinical use. Transversal as well as longitudinal images of the living eye have been acquired in less than $1 \mathrm{~s}$. This frame rate was obtained by sacrificing transversal resolution and by driving the galvo-scanner at a speed such that $20 \%$ of the line has to be discarded owing to the scanner flyback. The main limitation to acquisition speed is the galvo-scanner and the bandwidth of the balanced detection receiver.

Two modulation procedures are used. For small transversal images, where the image bandwidth is less than $15 \mathrm{kHz}$, phase modulation is introduced by a piezocylinder. For larger sizes, we demonstrate theoretically and experimentally that the path modulation introduced by the galvo-scanner itself can be used to obtain both longitudinal and transversal OCT images.

Even at a 0.85-s frame rate, eye movement still remains an issue for quantitative measurements. Building a 3-D profile from multiple stacks of transversal images taken at different depths appears to 
be very difficult. The procedure employed consisted of collecting a large number of images and discarding those where the eye movement disturbances were too large.

The transversal images demonstrate the potential of OCT to section the retina with very high depth resolution. Images taken at $50-\mu \mathrm{m}$ steps show noticeable differences. However, to take advantage of this resolution, further reduction of the acquisition time is necessary and the patient's head must be firmly fixed. Longitudinal imaging at a speed of $1 \mathrm{~mm} / \mathrm{s}$ was easily achieved, with no difficulty encountered in positioning the head and the eye to obtain an image.

As far as the authors are aware, this article represents the first reported comparison of transversal and longitudinal images produced using OCT.

\section{Acknowledgments}

The authors acknowledge the support of the UK Engineering and Physical Sciences Research Council and the referees' suggestions.

\section{REFERENCES}

1. D. Huang, E. A. Swanson, C. P. Lin, J. S. Schuman, W. G. Stinson, W. Chang, M. R. Hee, T. Flotte, K. Gregory, C. A. Puliafito, and J. G. Fujimoto, "Optical coherence tomography," Science 254, 1178 (1991).

2. W. V. Sorin, D. M. Baney, and S. A. Newton, "Optical lowcoherence reflectometry with $-148 \mathrm{~dB}$ sensitivity at 1.55 $\mu \mathrm{m}, "$ Proceedings of the 8th Optical Fiber Sensors Conference (IEEE/LEOS, Monterey, California, Jan. 29-31, 1992), pp. PD7, 1-4 (1993).

3. X. Clivaz, F. Marquis-Weible, R. P. Salathe, R. P. Novak, and H. H. Gilgen, "High resolution reflectometry in biological tissues," Opt. Lett. 17(1), 4-6 (1992).

4. B. Bouma, D. J. Tearney, S. A. Boppart, M. R. Hee, M. E. Brezinski, and J. G. Fujimoto, "High resolution optical coherence tomographic imaging using a mode-locked TiAl203 laser source," Opt. Lett. 20(13), 1486-1488 (1995).

5. X. J. Wang, T. E. Milner, R. P. Dhond, W. V. Sorin, S. A. Newton, and J. S. Nelson, "Characterization of human scalp hairs by optical low-coherence reflectometry," Opt. Lett. 20(6), 524-526 (1995).

6. A. Gh. Podoleanu, G. M. Dobre, D. J. Webb, and D. A. Jackson, "Simultaneous en-face imaging of two layers in human retina," Opt. Lett. 22(13), 1039-1041 (1997).

7. Hai-Pang Chiang and J. Wang, "Imaging through animal tissues with CW diode laser based broadband interferometry," Opt. Commun. 130, 317-326 (1996).

8. G. J. Tearney, B. E. Bouma, S. A. Boppart, B. Golubovic, E. A. Swanson, D. Huang, and J. G. Fujimoto, "Rapid acquisition of in vivo biological images by use of optical coherence tomography," Opt. Lett. 21(17), 1408-1410 (1996).

9. A. M. Sergeev, V. M. Gelikonov, G. V. Gelikonov, F. I. Feldchtein, N. D. Gladkova, and V. A. Kamensky, "Biomedical diagnostics using optical coherence tomography," TOPS Vol. 2, Advances in Optical Imaging and Photon Migration, R.
R. Alfano and J. G. Fujimoto, eds., Orlando, Florida, Optical Society of America, p. 196 (1996).

10. A. F. Fercher, "Optical coherence tomography," J. Biomed. Opt. 1(2), 157-173 (1996).

11. A. Gh. Podoleanu, G. M. Dobre, D. J. Webb, and D. A. Jackson, "Fiberized set-up for eye length measurement," Opt. Commun. 137, 397-405 (1997).

12. A. F. Fercher, K. Mengedoht, and W. Werner, "Eye length measurements by interferometry with partially coherent light," Opt. Lett. 13, 186-189 (1988).

13. A. F. Fercher, C. Hitzenberger, and M. Juchem, "Measurement of intraocular optical distances using partially coherent laser light," J. Mod. Opt. 38, 1327-1333 (1991).

14. W. Drexler, H. Sattman, C. K. Hitzenberger, and A. F. Fercher, "Measurement of the thickness of fundus layers by partial coherence tomography," Opt. Eng. 34(3), 701-710 (1995).

15. C. A. Puliafito, M. R. Hee, C. P. Lin, E. Reichel, J. S. Schuman, J. S. Duker, J. A. Izatt, E. A. Swanson, and J. G. Fujimoto, "Imaging of macular diseases with optical coherence tomography,"' Opthalmology 102(2), 217-229 (1995).

16. E. A. Swanson, J. A. Izatt, M. R. Hee, D. Huang, C. P. Lin, J. S. Schuman, C. A. Puliafito, and J. G. Fujimoto, "In vivo retinal imaging by optical coherence tomography," Opt. Lett. 18(21), 1864-1867 (1993).

17. B. R. Masters, Noninvasive Diagnostic Techniques in Opthalmology, Springer-Verlag, New York (1990).

18. Data sheets of Humphrey Instruments, optical coherence tomography, Humphrey Instruments, 2992 Alvarado St., San Leandro, CA 94577, (1996).

19. R. H. Webb, "Scanning laser ophthalmoscope," Opt. Lett. 18(21), 438-450 (1993).

20. Data sheets of scanning laser ophthalmoscope, G. Rodenstock Instruments GmbH, Postfach 1326 (1996) and Data sheets of Heidelberg laser scanning systems, Heidelberg Engineering, GmbH, Heidelberg, Germany (1996).

21. F. W. Fitzke, H. Woon, G. Timberlake, L. Robinson, J. Marshall, and A. C. Bird, "Optical modifications to a scanning laser ophthalmoscope for high magnification, narrow optical section imaging," Laser Light Ophthalmol. 4(1), 7-14 (1991).

22. A. Gh. Podoleanu, G. M. Dobre, D. J. Webb, and D. A. Jackson, "Fiberised set-up for retinal imaging of the living eye using low coherence interferometry," IEE Colloq. on Progress in Fibre Optic Sensors \& their Applications, April 1997, pp. 12/1-12/5.

23. D. A. Jackson and J. D. C. Jones, "Interferometers," in Optical Fiber Sensors: Systems and Applications, B. Culshaw and J. Dakin, eds., Vol. 2, Chap. 10, p. 349, Artech House, Norwood MA (1989).

24. K. Takada, A. Himeno, and K. Yukimatsu, "Phase-noise and shot-noise limited operations of low coherence optical time domain reflectometry," Appl. Phys. Lett. 59(20), 2483-2485 (1991).

25. A. Gh. Podoleanu, G. M. Dobre, D. J. Webb, and D. A. Jackson, "Coherence imaging by use of a Newton rings sampling function," Opt. Lett. 21(21), 1789-1791 (1996).

26. C. B. Su, "Achieving variation of the optical path length by a few millimeters at millisecond rates for imaging of turbid media and optical interferometry: a new technique," Opt. Lett. 22(10), 665-667 (1997).

27. R. N. Weinreb, A. W. Dreher, and J. F. Bille, "Quantitative assessment of the optic nerve head with the laser tomographic scanner," Int. Ophthalmol. 13, 25-29 (1989). 